\title{
Angular momentum of an electric charge and magnetically charged black hole
}

\author{
David Garfinkle and Soo-Jong Rey* \\ Dept. of Physics, University of California, Santa Barbara, CA 93106 \\ *Institute for Fundamental Theory, University of Florida, Gainesville
}

\section{FL 32611}

\section{DISCLAIMER}

This report was prepared as an account of work sponsored by an agency of the United States Government. Neither the United States Government nor any agency thereof, nor any of their employees, makes any warranty, express or implied, or assumes any legal liability or responsibility for the accuracy, completeness, or usefulness of any information, apparatus, product, or process disclosed, or represents that its use would not infringe privately owned rights. Reference herein to any specific commercial product, process, or service by trade name, trademark, manufacturer, or otherwise does not necessarily constitute or imply its endorsement, recommendation, or favoring by the United States Government or any agency thereof. The views and opinions of authors expressed herein do not necessarily state or reflect those of the United States Government or any agency thereof.

\section{Abstract:}

We find the angular momentum $L$ of a point particle with electric charge $e$ held at a fixed position in the presence of a black hole with magnetic charge $g$. (For a point charge in the presence of an ordinary magnetic monopole, it is known that $L=e g$ ). The angular momentum does depend on the separation distance between the particle and the black hole; however, $L \rightarrow e g$ for a large separation. Implications for the cosmic censorship hypothesis. the quantum hairs and other physical situations are discussed. 


\section{INTRODUCTION}

One of the most remarkable consequences of the existence of magnetic monopoles is that it implies the quantization of electric charge. One simple way to see this is to calculate the angular momentum $L$ of a stationary point charge $e$ in the presence of a point monopole of magnetic charge $g$. The result is $L=e g$ where the angular momentum points from the charge to the monopole. ${ }^{1}$ Together with the quantization of angular momentum this result then implies that electric charge is quantized.

Most grand unified theories possess t'Hooft-Polyakov magnetic monopoles ${ }^{2}$. They are nonsingular, finite energy field configurations with nontrivial abelian magnetic charge. In general relativity there exists a different sort of magnetic monopole: a magnetically charged black hole. Even though the metric is singular at the origin, it is shielded by the event horizon. Therefore, this is regarded as a valid classical field configuration. This is in fact a magnetic analog of the electrically charged black hole described by the Reissner-Nordstrom metric $^{3}$, and is related to it by a duality transformation. It is known that a magnetic monopole with mass greater than $(\alpha G)^{-1 / 2}$ forms a magnetic black hole ${ }^{4}$. Even though these magnetic black holes evaporate initially, after reaching the extreme Reissner-Nordstrom limit (maximally charged magnetic black hole), they remain absolutely stable.

In this paper we examine the angular momentum of a point electric charge held in place in the presence of a magnetically charged black hole. This calculation is complicated by the fact that the black hole can posess an intrinsic spin in addition to the angular momentum that comes from the electric and magnetic charges. Therefore we must distinguish between these two contributions to the total angular momentum. In the next section we calculate the total angular momentum of the system. Surprisingly, we find that the total angular momentum depends on the separation distance between the black hole and the electric charge. This gives a possibility to spin up the black hole by moving the charge closer to it. However, in the extreme Reissner-Nordstrom black hole case, the angular momentum is precisely e $g$, so 
spinning up the black hole is not possible as long as the charge stays outside the horizon. Physical implications of our result are discussed in the last section.

\section{ANGUlar. MOMENTUM}

Let $M, g$ and $e$ be respectively the mass and magnetic charge of the black hole and the electric charge of the particle. We treat the case where $e$ is small and calculate the angular momentum to first order in $e / g$ and $e / M$. Then to zeroth order the spacetime is a black hole with magnetic charge and zero spin. The metric is the Reissner-Nordstrom metric ${ }^{3}$

$$
g_{a b}^{(0)}=-f d t^{2}+f^{-1} d r^{2}+r^{2}\left(d \theta^{2}+\sin ^{2} \theta d \phi^{2}\right)
$$

where

$$
f \equiv 1-\frac{2 G M}{r}+\frac{G g^{2}}{r^{2}} .
$$

The radius $r_{H}$ of the black hole horizon is

$$
r_{H}=G M+\sqrt{G^{2} M^{2}-G g^{2}},
$$

which attains its minimum size for the case of the extreme Reissner-Nordstrom black hole, $g^{2}=G M^{2}$.

The hole's electromagnetic field tensor is (in CGS units)

$$
F_{a b}^{(0)}=2 g \sin \theta \nabla_{[a} \theta \nabla_{b]} \phi .
$$

Let the point charge be located at $r=b, \theta=\pi$. then its current vector is

$$
j^{a}=\frac{e}{2 \pi b^{2} \sin \theta} \delta(r-b) \delta(\theta-\pi)\left(\frac{\partial}{\partial t}\right)^{a} .
$$

The system (the black hole plus particle) is stationary and axisymmetric and thus has the Killing fields $(\partial / \partial t)^{a}$ and $(\partial / \partial \phi)^{a}$. We want to solve to first order for this system the Einstein-Maxwell equations

$$
R_{a b}=G\left(2 F_{a c} F_{b}{ }^{c}-\frac{1}{2} g_{a b} F_{c d} F^{c d}\right),
$$




$$
\nabla^{a} F_{a b}=-4 \pi j_{b}
$$

(The stress-energy of the particle makes a contribution to Einstein's equation, but not to the angular momentum; so we neglect it). Actually we need only those components of the metric and Maxwell tensor that determine the angular momentum. The angular momentum $\mathcal{L}(r)$ contained within a two-sphere $\mathcal{S}$ of constant $r$ and $t$ is defined by

$$
\mathcal{L}(r) \equiv \frac{1}{16 \pi G} \int_{\mathcal{S}} \epsilon_{a b c d} \nabla^{c}\left(\frac{\partial}{\partial \phi}\right)^{d}
$$

Thus, the total angular momentum $L$ is given by $L=\lim _{r \rightarrow \infty} \mathcal{L}(r)$. Let us define the quantity $X, \chi$ and $Z$ by

$$
\begin{gathered}
X \equiv g_{t \phi}, \\
\chi \equiv \frac{3}{4} \int_{0}^{\pi} X \sin \theta d \theta, \\
Z \equiv r^{4} \frac{d}{d r}\left(\chi / r^{2}\right) .
\end{gathered}
$$

Then using equation (9) it is straightforward to show that

$$
L=\frac{1}{6 G} \lim _{r \rightarrow \infty} Z
$$

Thus to find the angular momentum we need only to determine $Z$.

We now find an equation for $Z$. From the Bianchi identity $\nabla_{[a} F_{b c]}=0$ and the fact that the Lie derivative of $F_{a b}$ with respect to $(\partial / \partial t)^{a}$ vanishes, it follows that there is a scalar $U$ such that

$$
F_{t a}=\nabla_{a} U
$$

Define the quantity $u$ by

$$
u \equiv \frac{3}{2} \int_{0}^{\pi} U \sin \theta \cos \theta d \theta .
$$

Taking the $t-\Phi$ component of Einstein's equation and the $t$ component of Maxwell's equation and evaluating all quantities to first order, we find the following equations for $u$ and $\chi$.

$$
f \frac{d^{2} \chi}{d r^{2}}-\frac{2}{r^{2}}\left(f+\frac{2 G g^{2}}{r^{2}}\right) x+\frac{4 G g}{r^{2}} u=0 \text {, }
$$




$$
\frac{f}{r^{2}} \frac{d}{d r}\left(r^{2} \frac{d u}{d r}\right)-\frac{2}{r^{2}} u+\frac{2 g}{r^{4}} x=6 \pi \int_{0}^{\pi} j_{t} \sin \theta \cos \theta d \theta
$$

Boundary conditions and equations (11) and (15) allow us to express $\chi$ and $u$ in terms of $Z$. Equation (16) then leads to the following equation for $Z$.

$$
\frac{d}{d r}\left[r^{2} \frac{d}{d r}\left(f \frac{d Z}{d r}\right)-2\left(1+\frac{2 G g^{2}}{r^{2}}\right) Z\right]=-\frac{24 \pi G g r^{2}}{f} \int_{0}^{\pi} j_{t} \sin \theta \cos \theta d \theta
$$

The term on the right and side of equation (17) vanishes when $r \neq b$. Thus for all values of $r$ other than $b$ the furction $Z$ is a solution of the homogeneous equation. The three solutions of the homogeneous equation are

$$
\begin{gathered}
Z_{1}=r^{2} \\
Z_{2}=6-\frac{4 g^{2}}{M r} \\
Z_{3}=3 A(r) \int_{r}^{\infty} \frac{d \tilde{r}}{f(\tilde{r}) A^{2}(\tilde{r})} .
\end{gathered}
$$

where the function $A(r)$ is given by

$$
A(r) \equiv r^{2}-3 G g^{2}+\frac{2 G g^{4}}{M r}
$$

We find that $A(r)$ is monotonically increasing function of $r$ for $r>r_{H}$ and vanishes at $r=r_{H}$ for the extreme Reissner-Nordstrom black hole case. Furthermore, the quantity $Z_{1}$ is badly behaved as $r \rightarrow \infty$ and $Z_{3}$ is badly behaved as $r \rightarrow r_{H}$. It then follows that $Z$ is given by

$$
\begin{array}{ll}
Z=k_{1} Z_{1}+k_{2} Z_{2}, & r<b \\
Z=k_{3} Z_{2}+k_{4} Z_{3}, & r>b
\end{array}
$$

wheie $k_{1}, \cdots, k_{4}$ are constants.

It follows from equations (18c) and (19) that $\lim _{r \rightarrow \infty} r Z_{3}=1$. Then using equations (12) and (20b) we find $L=k_{3} / G$. We now have to separate out the intrinsic spin of the black hole. In order to insure that none of this angular momentum is due to intrinsic spin of the 
black hole we impose the condition that there is no angular momentum contained within the black hole's event horizon: that is $\mathcal{L}\left(r_{H}\right)=0$. Using equation (20a) this condition becomes

$$
k_{1} Z_{1}\left(r_{H}\right)+k_{2} Z_{2}\left(r_{H}\right)=0
$$

It follows from equation (17) that $Z$ and $d Z / d r$ are continuous at $r=b$. Using equations (5) and (17) we find that the discontinuity in $d^{2} Z / d r^{2}$ is given by

$$
\left[\frac{d^{2} Z}{d r^{2}}\right]_{b-\epsilon}^{b+\epsilon}=-\frac{12 G e g}{b^{2} f(b)}
$$

Equations (21) and (22) along with the continuity of $Z$ and $d Z / d r$ then yield four linear equations for the quantities $k_{1}, \cdots, k_{4}$. Some straightforward but tedious algebra then leads to the following expression for the angular momentum:

$$
L=e g\left[1-\frac{A\left(r_{H}\right)}{A(b)}\left(\frac{r_{H}}{b} \frac{3 M b-2 g^{2}}{3 M r_{H}-2 g^{2}}-2 Z_{3}(b) f(b) \frac{G M b-G g^{2}}{2 r_{H}-G M}\right)\right]
$$

Equation (23) is the main result of this paper. Most importantly, the angular momentum $L$ does depend upon the separation distance $b$. However, in the extreme Reissner-Nordstrom black hole, $L=e g$ still because $A\left(r=r_{H}\right)$ vanishes identically.

\section{Discussions}

1. The total angular momentum $L$ in Eq.(23) consists of two contributions. The first term in Eq.(23) is due to the electromagnetic field (i.e., the electric chargs and magnetic monopole contribution as in flat spacetime). The second term comes from the modification due to the gravitational interaction. The electromagnetic energy-momentum tensor modifies the metric, and similarly the angular momentum. This is clearly seen if we consider the limit $G \rightarrow 0$. Then, from Eq.(3), the horizon $r_{H}$ goes to zero as expected. Using this fact, we find the second term in Eq.(23) vanishes as we turn off the gravity. 
A similar simplification arises when the separation distance $b \rightarrow \infty$ : we have $L \rightarrow e g$, the same value as for an ordinary magnetic monopole plus a charged particle. Keeping the next leading term, we find

$$
L \rightarrow e g\left(1-\frac{2 r_{H}}{b^{2}} \frac{\left(G^{2} M^{2}-G g^{2}\right)}{\left(2 r_{H}-G M\right)}\right) \quad \text { as } b \rightarrow \infty .
$$

It also follows from equation (23) that $L \rightarrow 0$ as $b \rightarrow r_{H}$. By differentiating equation (23) with respect to $b$ one can show that $L$ is a monotonic function of $b$. Thus $L$ changes monotonically from 0 to $e g$ as $b$ goes from $r_{H}$ to $\infty$.

2. The quantity $L$ is the angular momentum that the system has when the black hole carries no spin. However, in general, the black hole can have an intrinsic spin. We may define the spin of black hole as the angular momentum inside the event horizon.

$$
S=\frac{1}{16 \pi G} \int_{r=r_{H}} \epsilon_{a b c d} \nabla^{c}\left(\frac{\partial}{\partial \phi}\right)^{d} .
$$

Then, the total angular momentum $\vec{J}=\vec{L}+\vec{S}$ is conserved. Now, let us imagine lowering the electrically charged particle into the magnetically charged $b^{\prime}$ ack hole. Since the system is axisymmetric, the total angular momentum $J$ is conserved. However, since $L$ depends on the position of the particle, it follows that the intrinsic spin of the black hole changes in the lowering process. Since $L$ is a monotonic function of $b$, the intrinsic spin of the black hole increases monotonically as the charge is lowered. Thus lowering the charge spins up the black hole.

In a system consisting of the 't Hooft-Polyakov monopole and a charge with nonzero isospin $\vec{I}$, it is known that $\vec{K}=\vec{S}+\vec{I}$ is conserved. Thus, the isospin degrees of freedom of the charge field can be transmuted into the spin of the monopole-charge composite ${ }^{5}$; if we turn on the gravity to convert the monopole into the magnetic black hole, since the inner core of the monopole is completely collapsed down to a singularity, we do not have such a possibility. Instead, we have a novel mechanism of angular momentum transmutation from the charge to the spin of the black hole which is not possible for the monopole-charge system. 
This transmutation of angular momentum also raises an intriguing question regarding the cosmic censorship hypothesis. The mass $M$, the electric and the magnetic charges $e, g$, and the angular momentum $J$ of a black hole must satisfy an inequality

$$
M^{2} \geq(J / M)^{2}+\left(e^{2}+g^{2}\right)
$$

otherwise the black hole disappears, leaving behind a naked singularity. The extreme (Kerr) Reissner-Nordstrom black hole saturates this inequality. Therefore if one could increase the charge or angular momentum of an extreme Reissner-Nordstrom black hole without changing its mass; then one could turn a black hole into a naked singularity; thus producing a counterexample to cosmic censorship. Thought experiments to turn black holes into naked singularities have been analyzed and in all cases studied cosmic censorship holds. ${ }^{6,7}$ In our case for the extreme Reissner-Nordstrom black hole $L=e g$ for all values of the separation between charge and black hole. Thus we cannot spin up the black hole by lowering the charge toward the horizon. One could change both the angular momentum and the charge of the black hole by lowering the charge through the horizon. We calculated all quantities to first order in $e$ and the contributions to the inequality are second order in $e$. Therefore our calculation does not resolve completely the issue of whether cosmic censorship can be violated by lowering the charge into the black hole.

3. A similar phenomenon may well arise within the pure gravity. In gravity, the analog of the electric and the magnetic charges are the mass $M$ and the 'dual' mass $N$. This can be understood, for example, from the analysis of the post-Newtonian limit of general relativity ${ }^{8}$. The metric solution that carries both the mass and the 'dual' mass (therefore, a proper 'gravitational dyon') is a cover of the Taub-NUT metric ${ }^{9}$ with a topology $S^{2} \times R^{2}$. An analug of the electric-magnetic duality transformation is the Geroch transformation 10 in the mass-'dual' mass plane of the Taub-NUT solution. Furthermore, it is known that the 'dual' mass $N$ has a topological origin, and is thus exactly conserved ${ }^{11}$. From all these analogies, if a point particle of mass $m$ is placed in the Taub-NUT background, we expect 
that the system acquires an angular momentum similar to Eq.(23) with $e \rightarrow m$ and $g \rightarrow N$ , in analogy with the electric charge-magnetic black hole system.

4. Finally let us imagine that the black hole and charge system with total angular momentum $J$ is studied in the Higgs phase of the $U(1)$ theory in which the condensate carries electric charge $n e(n=$ integer $)$. The original $U(1)$ symmetry is now broken down to $Z_{n}$. This situation raises an apparent puzzle: the field strength decays exponentially away from the sources. Thus, there seems to exist no field angular momentum associated with the electric charge and magnetic black hole in the Higgs phase. However, the Higgs condensate is not expected to break the Lorentz invariance, and in particular, the rotational invariance. Therefore, even after the vacuum is changed from the Coulomb to the Higgs phase, the (separation-dependent) angular momentum ought to be conserved. In fact, even though the classical electric field decays exponentially from the charge, there still remains a large fluctuations of quantum mechanical electric field that can be detected through the AharonovBohm interference ${ }^{12}$. Being a quantum hair, it cannot be exting rished even behind the black hole horizon. We suspect that the Aharonov-Bohm interaction gives rise to the quantum mechanical field angular momentum; however, we have not explicitly calculated this effect.

\section{ACKNOWLEI)GMENT}

We would like to thank Abhay Ashtekar, Joshua Frieman, James B. Hartle, Gary 1 Horowitz, Ted Jacobson, Pierre Sikivie, Jonathan Simon, Charles B. Thorn and Robert M. Wald for helpful discussions. Part of this work was done at the Aspen Center for Physics during the summer of 1990. This work was supported in part by NSF Grant No. PHY-8506686 and PHY-90-08502 (DG and SJR) to The University of California and DOE Grant No. DE-FG05-86ER-40272 (SJR) to The University of Florida. 


\section{REFERENCES}

${ }^{1}$ J. Jackson, Classical Electrodynamics, Chap.6 (Wiley, New York, 1975) .

${ }^{2}$ G. 't Hooft, Nuclear Physics B79(1974) 276; A.M. Polyakov, JETP Letters, 2o(1974) 194

; for a review, S. Coleman, 'The Magnetic Monopole Fifty Years Later', lecture at Erice School of Subnuclear Physics (1981).

${ }^{3}$ D. Kramer, H. Stephani, E. Herlt and M. MacCallum, Exact Solutions of Einstein's Fieid Equations, Cambridge University Press, Cambridge, England, (1980).

${ }^{4}$ W.A. Hiscock, Phys. Rev. Lett. 5o(1983) 1734; J. Frieman and C.T. Hill, SLAC-PUB-4283 (1987) unpublished.

${ }^{5}$ R. Jackiw and C. Rebbi, Phys. Rev. Lett. 36(1976) 1116; P. Hasenfratz and G. 't Hooft, Phys. Rev. Lett. 36(1976) 1119.

${ }^{6}$ R. M. Wald, Ann. Phys. 82(1974) 548.

${ }^{7}$ I. Semiz, Class. Quantum Grav. 7(1990) 353.

${ }^{8}$ A. Zee, Phys. Rev. Lett. 55(1985) 2379; for a fully general relativistic description, see Y.M. Cho, Theory of Gravitational Monopole, SNUITP 90-01 preprint (1990).

${ }^{9}$ E.T. Newman, L. Tamburino, and T. Unti, J. Math. Phys. 4(1963) 915; C.W. Misner, J. Math. Phys. 4(1963) 924; J. Dowker, Gen. Rel. Grav. 5(1974) 603.

${ }^{10}$ R.O. Hanson, J. Math. Phys. 15(1974) 46.

${ }^{11}$ A. Aslitekar and A. Sen, J. Math. Phys. 23(1982) 2168.

${ }^{12}$ L.M. Krauss and F. Wilczek, Phys. Rev. Lett. 62(1989) 1221: J.P. Preskill and L.M. Krauss, Nucl. Phys. B341(1990) 50: J.P. Preskill, Quantum Hair, CALT-68-1671 preprint (1990). 

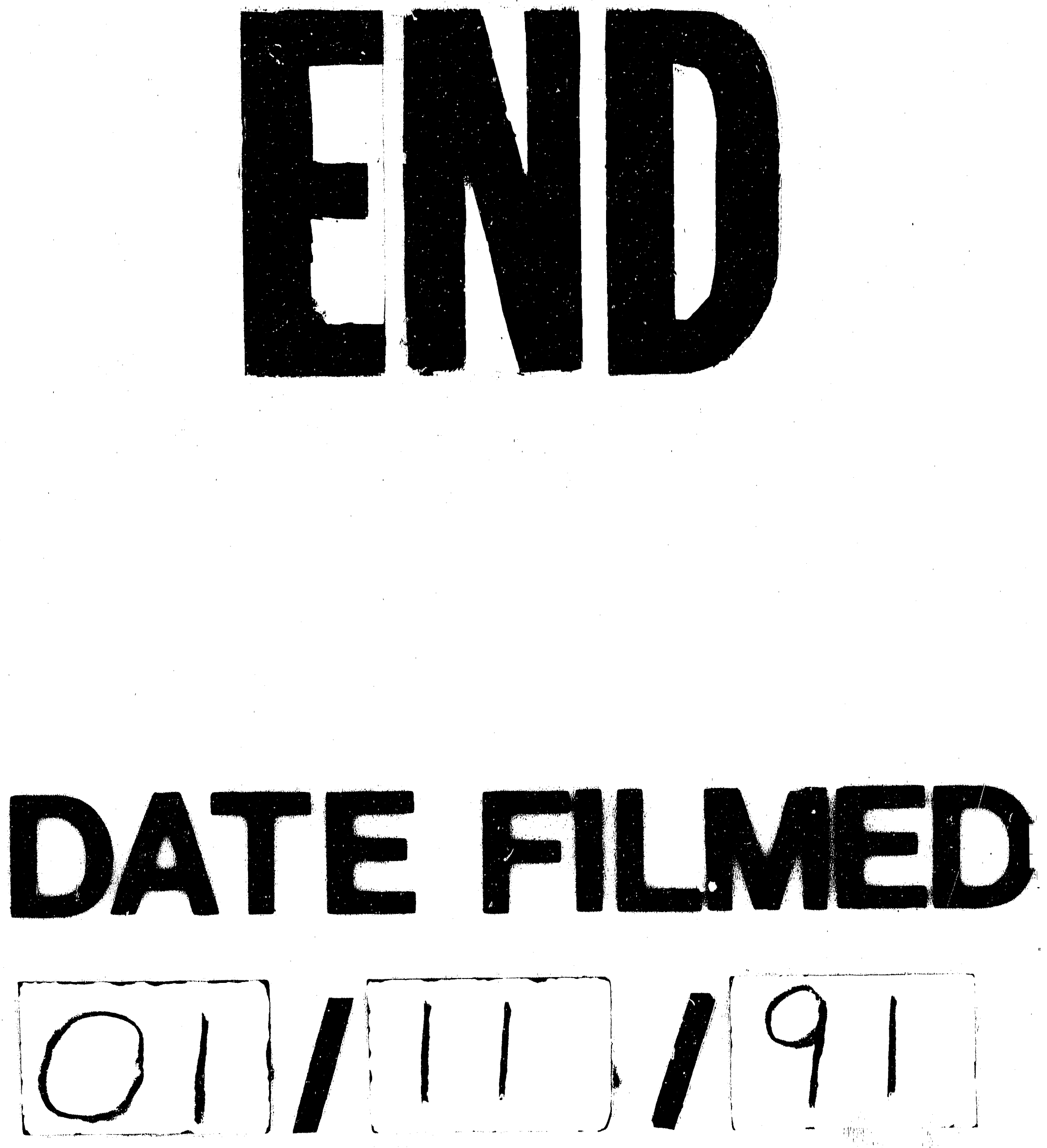
